\title{
The Effects of Myo-inositol on Glucose Metabolic in Diabetes Rats
}

\author{
Yin Xue-ping1,2, Guo Fei-fei", Sun Xiangrong ${ }^{1}, \mathrm{Xu} \mathrm{Luo}^{1}$
}

\author{
${ }^{1}$ Dept. of Pathophysiology, Medical College of Qingdao University, Qingdao, Shandong, 266021, China \\ ${ }^{2}$ Gaomi City People's Hospital, Gaomi, Shandong, 261500, China
}

\begin{abstract}
Objective: To observe the myo-inositol on normal and diabetic rats muscle and intestinal glucose uptake. Methods: The rat jejunum was placed in Kb with containing 2.5\% -20\% myo-inositol and the glucose concentration was detected at 0 and $2 \mathrm{~h}$. The psoas muscle was placed in $\mathrm{Kb}$ containing $2.5 \%-20 \%$ myo-inositol $\mathrm{Kb}$ (with or without insulin) the glucose concentration was detected at 0 and $1 \mathrm{~h}$. Rats were divided into blank control group, normal muscle myo-inositol group, blank control group of diabetic rats, myo-inositol group of diabetic rats and acarbose group of diabetic rats (DBA) The intestinal propulsion rate and intestinal absorption of glucose in each group were detected. Results: In vitro studies found that myo-inositol can dose-dependently stimulate the absorption of glucose in the jejunum and promote the uptake of glucose by the psoas muscle in a dose-dependent manner. Myo-inositol can delay gastric emptying in diabetic rats, inhibit intestinal absorption of glucose, reduce postprandial blood glucose in diabetic rats and promote the rate of intestinal propulsion in diabetic rats. Conclusion: Myo-inositol can inhibit the intestinal absorption of glucose in glucose rats and promote the glucose uptake in muscle of normal rats and diabetic rats.
\end{abstract}

Keywords: Inositol, Diabetes, Glucose Metabolic

\section{Introduction}

There are two major types of diabetes type I and II, of which type 2 diabetes (T2D) is the most common type of diabetes, accounting for more than $90 \%$ of all cases of diabetes ${ }^{[1]}$ 。 Sedentary, high-fat and high-sugar diets, and high intakes of refined sugars and monosaccharides keep the body in high blood sugar for a long time ${ }^{[2]}$ 。For the treatment of diabetes, in addition to conventional antidiabetic drug therapy to reduce high-calorie and high-sugar food intake, and the combination of drugs inhibiting an increase in blood sugar after a meal has become more comprehensive diabetes treatment programs ${ }^{[3]}$ 。

Myo-inositol, a less used sugar alcohol, is similar in structure to glucose and is found in a variety of foods, but at lower concentrations ${ }^{[4]}$ 。D-inositol is another high content of inositol in fat, muscle and liver ${ }^{[5]}$ 。 Myo-inositol with glucose transporter activation and glucose utilization, D-inositol is mainly involved in the synthesis of glycogen in liver, fat and muscle tissue ${ }^{[4]}$ 。 Studies have also found that supplementation with myo-inositol can regulate glucose and lipid metabolism, the glucose and lipid metabolism are closely related to the pathogenesis of T2D. ${ }^{[6]}$ 。
At present, it is not yet clear whether muscle myo-inositol can affect glucose uptake by muscles, nor does research clearly indicate that myo-inositol can affect intestinal absorption of glucose. Therefore, this study investigated the effects of muscle inositol on glucose and glucose absorption in muscle and intestine of normal and diabetic rats through in vitro and in vivo studies.

\section{Materials and Methods \\ 2 Animals}

7-week-old male Sprague-Dawley rats were $184.20 \pm 5.22 \mathrm{~g}$, room temperature was controlled at $25 \pm 2{ }^{\circ} \mathrm{C}$, and day and night cycling light was used for $12 \mathrm{~h}$, and the diet was free. All animals followed the "Qingdao University Laboratory Animal Protection and Use Management Methods." All experiments were in accordance with the Qingdao Experimental Animal Center.

\subsection{In vitro studies}

5 rats were randomly selected and sacrificed after fasting for $12 \mathrm{~h}$. The entire gastrointestinal tract (GIT) and psoas muscles were dissected and used for glucose absorption and uptake studies. 


\subsubsection{Absorption of glucose by jejunum}

5 jejunum of $5 \mathrm{~cm}$ in length were randomly selected from the gastrointestinal tract of each rat and placed in $8 \mathrm{ml}$ of $\mathrm{Kb}$ fluid $(118 \mathrm{mM} \mathrm{NaCl}, 5 \mathrm{mM} \mathrm{KCl}, 1.328$ $\mathrm{mM} \mathrm{CaCl}_{2} \cdot 2 \mathrm{H}_{2} \mathrm{O}, 1.2 \mathrm{mM} \mathrm{KH}_{2} \mathrm{PO}_{4}, 1.2 \mathrm{mM} \mathrm{MgSO}_{4}$ 和 $25 \mathrm{mM} \mathrm{NaHCO}_{3}$ ) was filled with a mixture of $5 \%$ $\mathrm{CO}_{2}$ and $95 \% \mathrm{O}_{2}$, each containing $11.1 \mathrm{mM}(199.98$ $\mathrm{mg} / \mathrm{dL}$ ) Glucose was placed at a constant temperature of $37^{\circ} \mathrm{C}$.

\subsubsection{Absorption of glucose by psoas muscle}

12 rat psoas muscles were weighed at random, each 0.5 $\mathrm{g}$, divided into insulin $(200 \mathrm{mU} / \mathrm{mL})$ group and no insulin group $(\mathrm{n}=6)$, each component was muscle myo-inositol $(2.5 \%, 5 \%, 10 \%, 20 \%)$, metformin (2 $\mathrm{mg} / \mathrm{mL}$ ) and placebo, each psoas muscle was placed in $8 \mathrm{ml} \mathrm{Kb}$ solution containing $11.1 \mathrm{mM}(199.98 \mathrm{mg} / \mathrm{dL})$ glucose, filled with $5 \% \mathrm{CO}_{2}$ and A mixture of $95 \% \mathrm{O}_{2}$, insulin solution containing $200 \mathrm{mU} / \mathrm{mL}$ insulin in the $\mathrm{Kb}$ solution, each group were placed at $37{ }^{\circ} \mathrm{C}$ constant temperature experiments, and before the test and $1 \mathrm{~h}$ after the start of the test kit to detect $\mathrm{Kb}$ The concentration of glucose in the liquid.

\subsection{In vivo studies}

\subsubsection{Diabetic rat model preparation}

Diabetic rats were given $10 \%$ fructose solution to induce insulin resistance in rats, and normal rats were given normal drinking water. $40 \mathrm{mg} / \mathrm{kg}$ streptozotocin was injected intraperitoneally into diabetic rats, whereas in normal rats, citrate buffer was only injected intraperitoneally. One week after intraperitoneal injection, the rats' postprandial blood glucose was measured. Rats with postprandial blood glucose $>300$ $\mathrm{mg} / \mathrm{dl}$ were selected as diabetic rats for study.

\subsubsection{Rat grouping}

12 normal rats were randomly selected and divided into 2 groups $(n=6)$, normal rats blank control group (NC), and normal rat muscle myo-inositol group (NMI). 18 diabetic rats were randomly selected and divided into 3 groups $(n=6)$, diabetic control group (DBC), diabetic myo-inositol group (DMI), diabetic rat acarbose group (DBA).

The fasting blood glucose was measured after overnight fasting in rats. Rats in NMI and DMI groups were orally administered with a mixture of myo-inositol (1 g/kg) and glucose $(2 \mathrm{~g} / \mathrm{kg})$. DBA rats were gavage the mixed of acarbose. (100 mg/Kg) and glucose $(2 \mathrm{~g} / \mathrm{kg})$. The rats in the $\mathrm{NC}$ and DBC groups were gavage glucose $(2 \mathrm{~g} / \mathrm{kg}) .0 .05 \%$ phenol red was present in all gavage fluids. All rats were anesthetized with sevoflurane for $1 \mathrm{~h}$ after gavage, blood samples were collected by cardiac puncture, and the rat gastrointestinal tract (GIT) was isolated. The kit was used to detect the glucose level in the blood samples of the rats. The GIT was quickly frozen in liquid nitrogen and retained at $-30^{\circ} \mathrm{C}$.

\subsubsection{Intestine advancing rate and intestinal glucose absorption}

The GIT was divided into 8 segments. The stomach and small intestine were divided into 4 segments. The cecum and the colon were divided into 2 segments. After each segment was weighed, the contents of each segment were measured by the kit to measure the glucose concentration, and each segment of the content and tissue was separated. After homogenization, 15000 rpm centrifuged for $30 \mathrm{~min}$. The supernatant was used to measure the absorbance at $620 \mathrm{~nm}$. The phenol red concentration in each gastrointestinal tract was determined by referring to the phenol red standard absorbance luminosity curve. Calculate the gastric emptying rate $(\%)$, the intestinal propulsion rate $(\%)$, and the glucose absorption index (GAI).

\subsection{Statistical analysis}

The data in the experiment was represented by $\mathrm{X}$ \pm SEM. The data was statistically analyzed using Prism 5.0. T-test or one-way ANOVA was used between the two groups. $\mathrm{P}<0.05$ was considered statistically significant.

\section{Result}

\subsection{In vitro studies}

2.1.1 Effect of Myo-Inositol on Absorption of Glucose in the Jejunum of Isolated Rats

In vitro studies found that myo-inositol inhibited glucose uptake in the jejunum of rats in a dose-dependent manner $(\mathrm{p}<0.05 \sim 0.001)$. Myo-inositol at a concentration of $10 \%$ achieved a maximum inhibitory effect. 


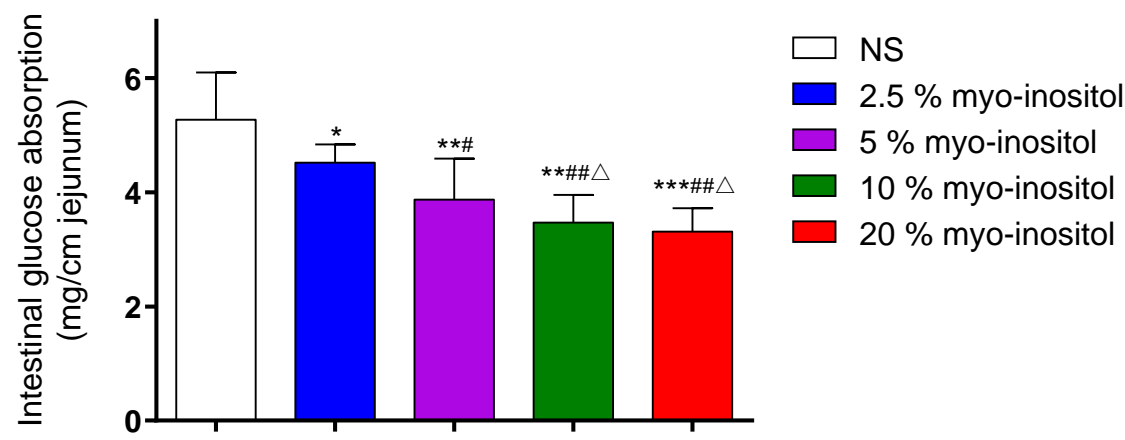

Fig. 1 Effect of Muscle Inositol on Absorption of Glucose in Jejunum of Rats ${ }^{*} \mathrm{p}<0.05, * * \mathrm{p}<0.01, * * * \mathrm{p}<0.001$, vs. NS group; ${ }^{\#} \mathrm{p}<0.05,{ }^{\#} \mathrm{p}<0.01$ vs. $2.5 \%$ myo-inositol group; ${ }^{\Delta} \mathrm{p}<0.05$ vs. $5 \%$ myo-inositol group

\subsubsection{Effect of Myo-Inositol on Glucose Uptake in Rat Psoas Muscle}

In vitro studies have shown that myo-inositol promotes glucose uptake in the psoas muscle in a dose-dependent

manner $(\mathrm{p}<0.05 \sim 0.001)$. This effect of myo-inositol promotes muscle uptake of glucose can be enhanced by insulin $(\mathrm{p}<0.05)$ 。

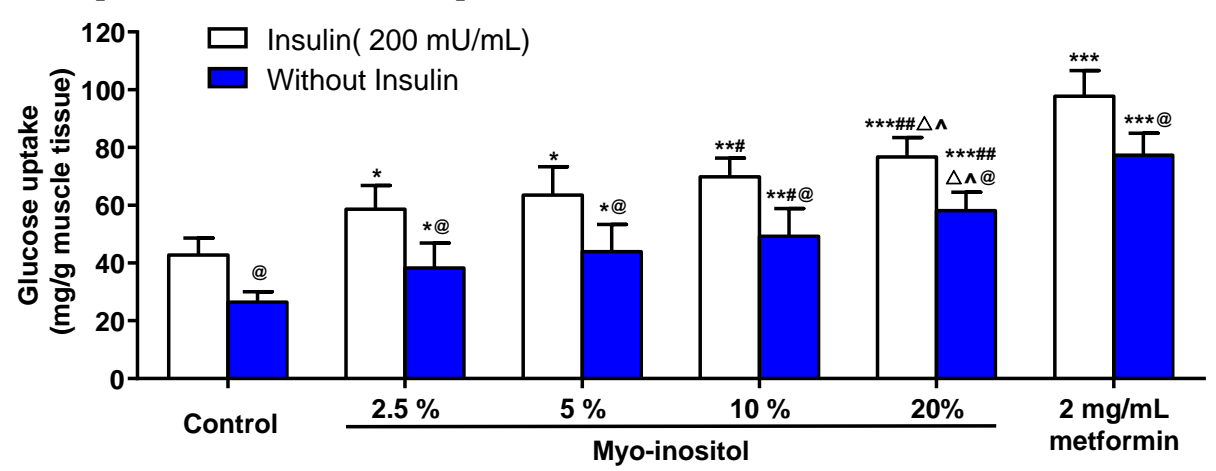

Fig. 2 Effect of Muscle Inositol on Glucose Uptake in Rat Psoas Muscle $* \mathrm{p}<0.05, * * \mathrm{p}<0.01, * * * \mathrm{p}<0.001$ vs. the control group with same insulin concentration; ${ }^{\#} \mathrm{p}<0.05,{ }^{\# \#} \mathrm{p}<0.01 \mathrm{vs}$. $2.5 \%$ myo-inositol with same insulin concentration; ${ }^{\Delta} \mathrm{p}<0.05$ vs. $5 \%$ myo-inositol with same insulin concentration; ${ }^{\wedge} \mathrm{p}<0.05$ vs. $10 \%$ myo-inositol with same insulin concentration

\subsection{In vivo studies}

\subsubsection{Effect of Myo-Inositol on Gastric Emptying}

Compared with diabetic rats given physiological saline, gastric emptying was decreased in diabetic with myo-inositol and acarbose rats $(\mathrm{P}<0.05)$, but there was no significant change in gastric emptying after administration of myo-inositol in normal rats.

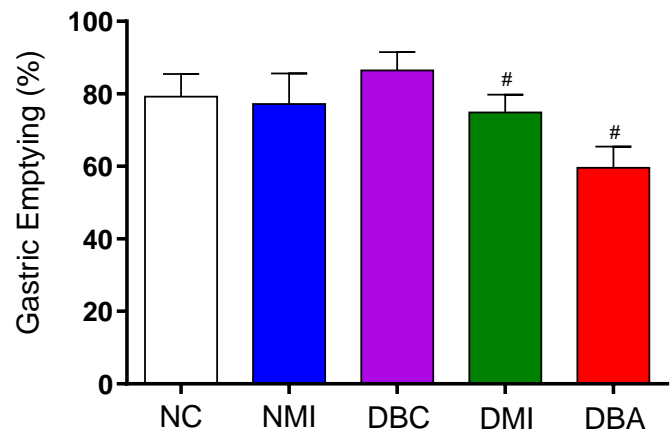

Fig. 3 Effect of Myo-Inositol on Gastric Emptying ${ }^{\#} \mathrm{p}<0.05$ vs. DBC group; $\hat{\mathrm{p}}<0.05$ vs. DMI group 
2.2.2 Effect of myo-inositol on intestinal absorption of glucose

In vivo studies found that myo-inositol can reduce glucose absorption in the first and second segments of the small intestine in normal rats $(\mathrm{p}<0.05)$, and in diabetic rats, glucose uptake in the first small intestine and cecum of diabetic rats after administration of myo-inositol $(\mathrm{p}<0.05)$. Acarbose inhibits glucose uptake in the first segment of the small intestine and the first segment of the colon in diabetic rats $(p<0.05)$ 。
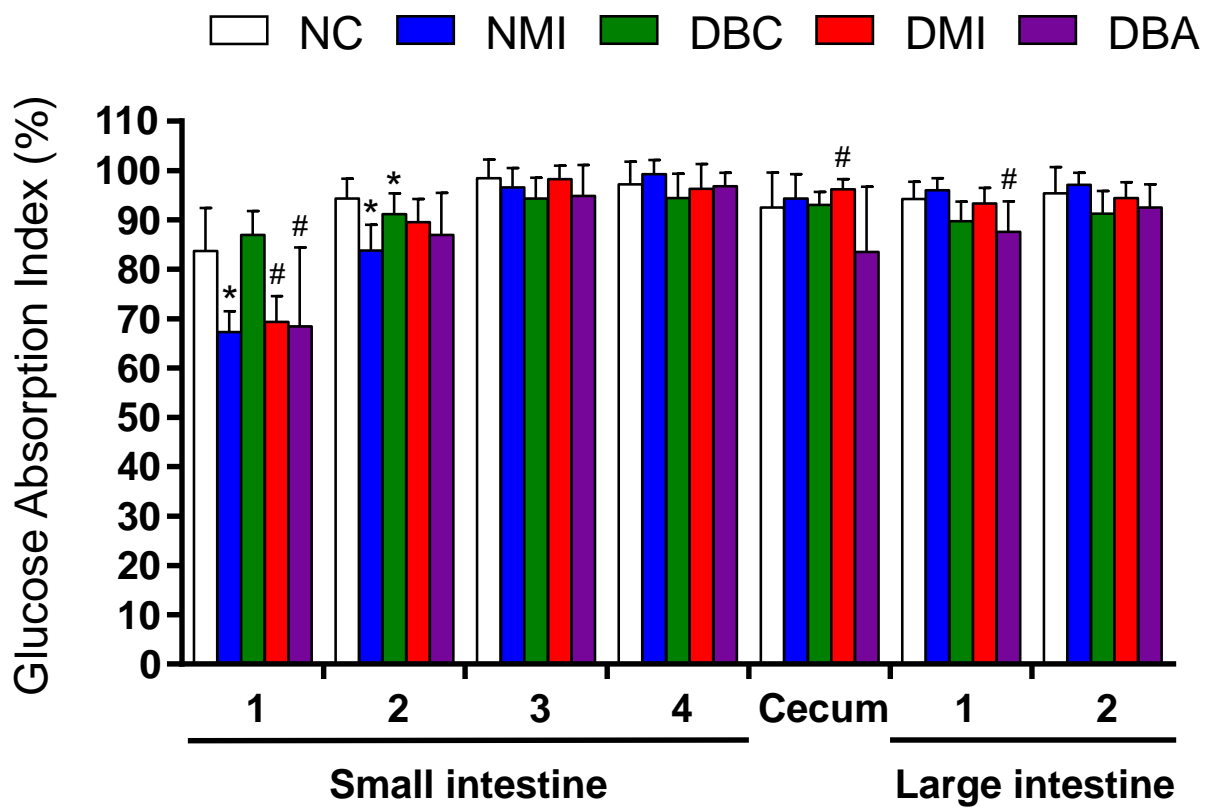

Fig. 4 Effect of myo-inositol on intestinal absorption of glucose ${ }^{*} \mathrm{p}<0.05$ vs. NC group; ${ }^{\mathrm{p}}<0.05$ vs. DBC group

\subsubsection{Effect of Myo-Inositol on Blood Glucose}

Myo-Inositol can not reduce blood glucose in normal rats $1 \mathrm{~h}$ after meal, but it can reduce the blood glucose level 1 hour after meal in diabetic rats. $(\mathrm{p}<0.05)$, Acarbose reduces blood glucose $1 \mathrm{~h}$ after meal in diabetic rats $(\mathrm{p}<0.05)$ 。

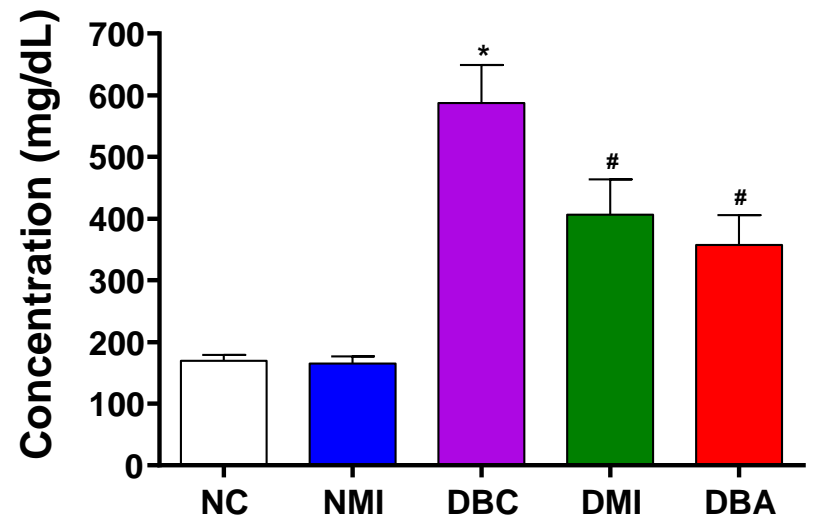

Fig. 5 Effect of Myo-Inositol on Blood Glucose ${ }^{*} \mathrm{p}<0.05$ vs. NC group; ${ }^{\mathrm{p}}<0.05$ vs. DBC group

\subsubsection{Effect of Myo-Inositol on Intestinal Propulsive} Rate

Myo-inositol improves intestinal propagation rates in the third, fourth, cecum and colon of normal and diabetic rats $(\mathrm{P}<0.05)$, and acarbose can only promote the intestinal propulsion rate of the first small intestine, cecum and colon in diabetic rats $(\mathrm{P}<0.05)$. 


\section{$\mathrm{NC} \square \mathrm{NMI} \square \mathrm{DBC} \square \mathrm{DMI} \square \mathrm{DBA}$}

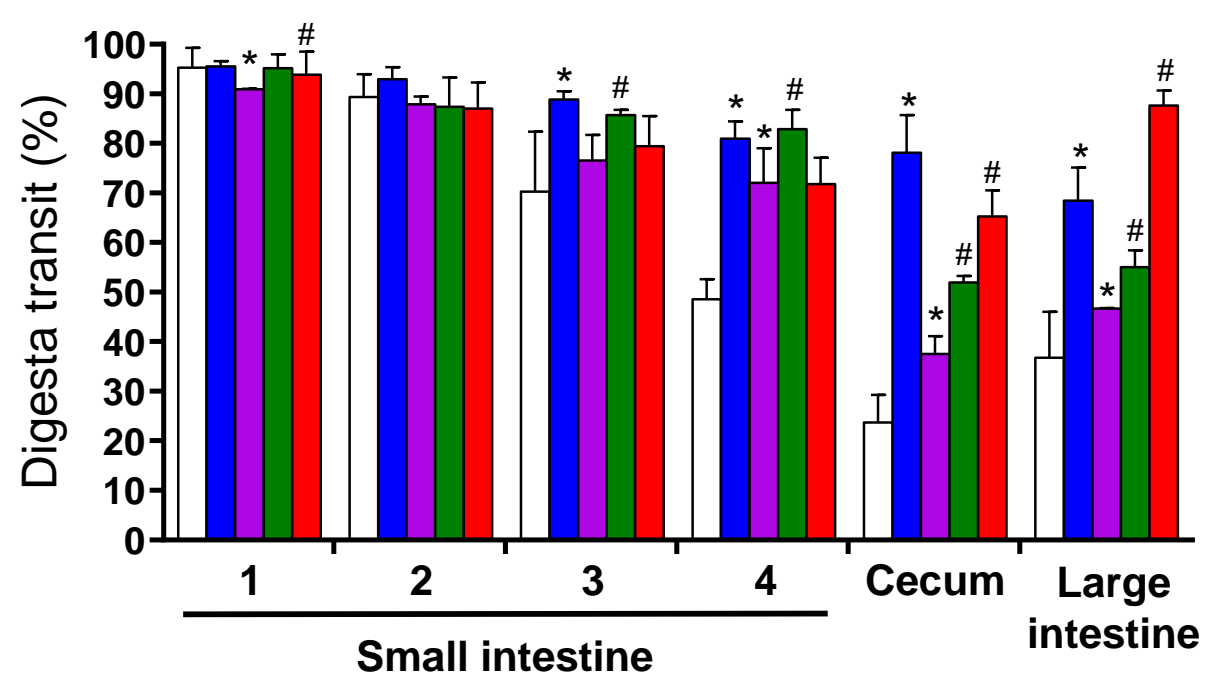

Fig. 6 Effect of myo-inositol on intestinal propulsive rate $* \mathrm{p}<0.05$ vs. NC group; ${ }^{\#} \mathrm{p}<0.05$ vs. DBC group

\section{Discuss}

Insulin enhances glucose metabolism to ensure stable blood sugar ${ }^{[7]}$, In patients with type 2 diabetes, insulin resistance or down-regulation of insulin sensitivity results in decreased glucose uptake by myocytes, lowering glucose tolerance in the body, and resulting in persistent hyperglycemia. ${ }^{[8]}$ 。

Since the uptake of glucose by muscle cells is an important indicator of insulin sensitivity, the effect of myo-inositol on glucose uptake in isolated rat psoas muscle (with or without insulin) was investigated in our study to investigate whether myo-inositol can improve the insulin sensitivity of diabetic patients. In this study, it was found that myo-inositol promoted the glucose uptake of isolated psoas muscle (with or without insulin) in a dose-dependent manner. It has been shown that at least in vitro, myo-inositol enhances glucose uptake by insulin-mediated muscles, but this finding is only ex vivo muscle and does not represent muscle regulation of glucose uptake in vivo. Supplementing myo-inositol may improve insulin sensitivity in somatic skeletal muscle ${ }^{[5,6]}$.

For glucose uptake, different segments of the small intestine have different glucose uptake ${ }^{[9]}$, therefore, we mainly observed the absorption of glucose in this part of the intestine during the study. In vitro studies found that myo-inositol inhibits the absorption of glucose in the intestine in a dose-dependent manner, indicating that myo-inositol has the potential to reduce postprandial blood glucose. In vivo study also found that normal rats, diabetic rats after the administration of myo-inositol in the first segment of the small intestine to glucose uptake, and to diabetic myo-inositol diabetic rats postprandial blood glucose increased significantly lower than Diabetic rats that were not given muscle myo-inositol. It was shown that myo-inositol may have the potential to inhibit the intestinal uptake of glucose in food.

Reduced gastric emptying and increased intestinal propulsion can reduce nutrient absorption and food intake in the intestine ${ }^{[10]}$, our study found that gastric emptying in diabetic rats increased, which is similar to the clinical manifestations of most patients with type 2 diabetes $^{[11]}$. This study found that gastric emptying in diabetic rats after intramuscular administration of myo-inositol was delayed, and both normal and diabetic rats showed an increase in the intestinal propulsion rate of the eel, which may be due to the similar structure of myo-inositol and glucose. It is able to competitively inhibit glucose, but this still needs further research to confirm.

Taken together, this study found that myo-inositol inhibits the absorption of glucose by the jejunum and increases glucose uptake by isolated muscles in vitro. Oral myo-inositol inhibits the absorption of glucose in the proximal small intestine and inhibits it. Gastric emptying increases the bowel intestine advancing rate. Although the results of this study found that oral myo-inositol can reduce postprandial blood glucose in diabetic rats, it is not entirely certain that this is due to myo-inositol inhibiting glucose absorption in the proximal small intestine. However, the results of the 
current study show that diabetic patients given a certain amount of myo-inositol can help control postprandial blood glucose, reduce intestinal glucose absorption, increase muscle glucose uptake, which will provide new drugs for clinical diabetes patients direction.

\section{References}

1. Kanamori H1, Takemura G, Goto K, et al. Autophagic adaptations in diabetic cardiomyopathy differ between type 1 and type 2 diabetes[J]. Autophagy. 2015, 11(7):1146-1160

2. Wu L, Piotrowski K, Rau T, et al. Walnut-enriched diet reduces fasting non-HDL-cholesterol and apolipoprotein $\mathrm{B}$ in healthy Caucasian subjects: a randomized controlled cross-over clinical trial[J]. Metabolism. 2014, 63(3):382-391

3. Azad MB, Abou-Setta AM, Chauhan BF, et al. Nonnutritive sweeteners and cardiometabolic health: a systematic review and meta-analysis of randomized controlled trials and prospective cohort studies[J]. CMAJ. 2017, 189(28):E929-E939

4. Duliński R, Stodolak B, Byczyński $Ł$, et al. Solid-State Fermentation Reduces Phytic Acid Level, Improves the Profile of Myo-Inositol Phosphates and Enhances the Availability of Selected Minerals in Flaxseed Oil Cake[J]. Food Technol Biotechnol. 2017, 55(3):413-419

5. Facchinetti F, Bizzarri M, Benvenga S, et al. Results from the international consensus conference on myo-inositol and D-chiro-inositol in obstetrics and gynecology: the link between metabolic syndrome and PCOS[J]. Eur J Obstet Gynecol Reprod Biol. 2015, 195:72-76

6. Dang NT, Mukai R, Yoshida K, et al. D-pinitol and myo-inositol stimulate translocation of glucose transporter 4 in skeletal muscle of C57BL/6 mice[J]. Biosci Biotechnol Biochem. 2010;74(5):1062-1067

7. Islam MS, Sakaguchi E. Sorbitol-based osmotic diarrhea: possible causes and mechanism of prevention investigated in rats[J]. World J Gastroenterol. 2006, 12(47):7635-7641

8. Cuthbert CE, Foster JE, Ramdath DD. A maternal high-fat, high-sucrose diet alters insulin sensitivity and expression of insulin signalling and lipid metabolism genes and proteins in male rat offspring: effect of folic acid supplementation[J]. Br J Nutr. 2017, 118(8):580-588

9. Röhe I, Boroojeni FG, Zentek J. Effect of feeding soybean meal and differently processed peas on intestinal morphology and functional glucose transport in the small intestine of broilers[[J]. Poult Sci. 2017, 96(11):4075-4084

10. Wölnerhanssen BK, Cajacob L, Keller N, et al. Gut hormone secretion, gastric emptying, and glycemic responses to erythritol and xylitol in lean and obese subjects[J]. Am J Physiol Endocrinol Metab. 2016, 310(11):E1053-E1061

11. Fukuhara S, Masaoka $\mathrm{T}$, Nishimura $\mathrm{S}$, et al. Enteric Glial Dysfunction Evoked by Apolipoprotein E Deficiency Contributes to Delayed Gastric Emptying[J]. Dig Dis Sci. 2017, 62(12):3359-3369 